\title{
Memoria, funciones ejecutivas y deterioro cognitivo en población anciana
}

\author{
Yolanda Clemente, Julia García-Sevilla e Inmaculada Méndez \\ Universidad de Murcia (España)
}

\begin{abstract}
Las funciones cognitivas hacen referencia a las funciones intelectivas o funciones mentales superiores. Suelen ser estos procesos los que primero sufren deterioro cognitivo asociado a la edad. El objetivo del presente estudio fue: analizar el estado de la memoria y las funciones ejecutivas en población anciana con y sin deterioro cognitivo; y comparar el estado cognitivo entre ancianos de un centro de personas mayores y ancianos en sus hogares. Se administró un test de screening para comprobar el nivel de deterioro cognitivo (Mini Examen Cognoscitivo MEC-35), para posteriormente, aplicar el Test de Alteración de Memoria (T@M) y el Test de las Anillas. Los participantes fueron 24 sujetos, de los cuales 12 pertenecían a un centro de personas mayores y 12 residían en sus hogares. La edad media de los participantes fue de 78 años. El estudio permitió determinar la relación significativa entre el nivel de deterioro cognitivo y el estado de la memoria y las funciones ejecutivas. El sexo fue indiferente en la variable memoria, pero no en funciones ejecutivas. Finalmente, el hecho de que la persona resida en una institución no mostró diferencias significativas frente a aquellos que residen en sus hogares en cuanto al estado de la memoria y las funciones ejecutivas.
\end{abstract}

Palabras clave: Funciones cognitivas, memoria, funciones ejecutivas, deterioro cognitivo.

Memory, executive functions and cognitive impairment in elderly population. Cognitive functions refer to the superior brain or mental function. These processes are usually first to suffer cognitive impairment associated with ageing. The aim of this study was: to analyse the state of memory and executive functions in a sample of elder population with and without cognitive impairment; and compare the cognitive state between elder who live in a public home for the elderly and elder who live in their homes. Therefore, a screening test was carried out to check the cognitive impairment level (Cognitive Mini Test MEC-35), and after that, the Memory Alteration Test (T@M) and the Ring Test were applied. The participants were 24 subjects, 12 of which belonged to a public home for the elderly and the other 12 lived in their homes. The average age of the participants was 78 . The study has allowed us to establish the significant relation between the cognitive deterioration level and the state of the memory and executive functions. Besides, sex was indifferent in memory as a variable, but not in executive functions. Finally, the fact of the people living in a public home show us no significant differences in the state of memory and executive functions compared to people who live in their homes.

Key words: Cognitive functions, memory, executive functions, cognitive impairment.

Correspondencia: Yolanda Clemente. Facultad de Psicología. Departamento de Psicología Básica y Metodología. Universidad de Murcia. Campus de Espinardo. C.P. 30100. Murcia (España). E-mail: juliags@um.es 
Actualmente se ha producido un incremento del envejecimiento en nuestra sociedad, el cual se debe fundamentalmente a los avances científicos y médicos y a las mejoras de la calidad de vida. Más concretamente, en nuestro país la proporción de personas mayores de 65 años en el año 2013 era del 17.7\%, según el Instituto Nacional de Estadística (INE, 2014). Por este motivo, cada vez nos encontramos con un interés mayor por comprender los efectos de la edad en el funcionamiento cognitivo.

En primer lugar, cuando hablamos de envejecimiento nos estamos refiriendo a un proceso natural. Se trata pues, de un proceso de duración variable, pero relativamente semejante para cada especie. Según Aller (2002), este consiste, principalmente en un incremento progresivo de la vulnerabilidad del organismo y de la probabilidad de padecer enfermedades.

Existen numerosas variables que modulan el proceso de envejecimiento, como las experiencias personales, el número de pérdidas significativas, los conocimientos alcanzados, las enfermedades padecidas, etc. Debido a esto, el proceso de envejecimiento resulta distinto en cada persona, por su historia personal y biológica, entre otras cosas.

Por otro lado, el concepto de funciones cognitivas hace referencia a las funciones intelectivas o funciones mentales superiores propias de la especie humana (memoria, pensamiento, conocimiento, planificación, lenguaje...). Además resulta importante destacar que, en el estado de las funciones cognitivas de las personas de la tercera edad, podemos encontrar una amplia variabilidad, debido a, entre otras cosas, el nivel educativo y la genética de la persona (factores ambientales y biológicos).

Cabe mencionar, además, la diferencia que se produce en lo que respecta al estado de las funciones cognitivas en un estado de envejecimiento normal y en caso de padecer deterioro cognitivo.

Una amplia variedad de estudios por parte de diferentes autores evidencian los cambios a nivel cognitivo asociados a la vejez, los cuales son, entre otros: enlentecimiento en el procesamiento cognitivo, alteración atencional (red de alerta, mecanismos de control inhibitorio y distraibilidad, especialmente), descenso de las capacidades ejecutivas, dificultades en algunos componentes del lenguaje (dificultad en el acceso léxico, procesamiento sintáctico y organización del discurso) y declive de algunos componentes de la memoria (verbal, visual y de trabajo, prioritariamente) (Brigman y Cherry, 2002; Myerson, Hale, Swagstaff, Poon, y Smith, 1990; Salthouse, 1996; Jennings, Dagenbach, Engle, y Funke, 2007; Valeriani, Ranghi, y Giaquinto, 2003; Vázquez et al., 2011; Binotti, Spina, de la Barrera, y Donolo, 2009; Hull, Martin, Beier, Lane, y Hamilton, 2008; Sorel y Pennequin, 2008; Juncos-Rabadán, Facal, Álvarez, y Rodríguez, 2006; Juncos-Rabadán, Pereiro, y Rodríguez, 2005; Thornton y Light, 2006; citados en García-Sevilla, Fernández, Fuentes, López, y Moreno, 2014). A 
este respecto, según Correia (2011), la memoria es una de las áreas cognitivas que más afectada se encuentra por el proceso de envejecimiento normal.

No obstante, una amplia variedad de capacidades continúan preservadas en el envejecimiento normal, las cuales son: gnosias, razonamiento verbal, memoria procedimental, memoria a largo plazo, diversos componentes del lenguaje (vocabulario, fonología, sintaxis y lenguaje escrito) y praxias.

Es necesario destacar que demencia y deterioro cognitivo son distintos, por lo que, toda demencia cursa con un deterioro cognitivo, pero no todo deterioro cursa con demencia. De este modo, el deterioro cognitivo es condición necesaria pero no suficiente para realizar un diagnóstico de demencia. Si explicásemos el deterioro cognitivo mediante el modelo del continuo cognitivo, podríamos decir que éste se encuentra en una fase intermedia, de modo que, en un extremo se encontraría la perfección cognitiva, y en otro, la demencia (González et al., 2001; Riquelme, Ortigosa, y Marín, 2010).

Pues bien, los criterios que cumplen los pacientes que tienen un deterioro cognitivo leve (DCL) (Aller, 2002; Riquelme et al., 2010) son los siguientes: 1) quejas de pérdida de memoria; 2) actividades de la vida diaria (AVD) normales; 3) función cognitiva general íntegra; 4) ejecución en memoria inferior a 1.5 desviaciones típicas o más por debajo de la media; y 5) No cumplen los requisitos para el diagnóstico de demencia.

Más concretamente, y atendiendo a los objetivos planteados en nuestro estudio, podemos decir que una de las funciones cognitivas más estudiadas en relación al envejecimiento es la memoria, puesto que, la mayoría de las quejas de memoria están asociadas a la edad. Las principales quejas de memoria suelen ser la dificultad para recordar nombres, números de teléfono o el lugar en el que se encuentran los objetos (fallos en la consolidación y transferencia de la información desde la memoria a corto plazo a la memoria a largo plazo). Además, las quejas y/o problemas relacionados con la memoria pueden afectar seriamente a la vida cotidiana de la persona mayor.

Muchos autores califican como normales los cambios en la memoria que se dan en el envejecimiento, pero es necesario saber que no todas las habilidades mnésicas se deterioran de igual forma con la edad, sino que la afectación se muestra distinta según los tipos de memoria. En relación a ello, se han realizado una serie de estudios que demuestran que las diferencias más significativas las podemos observar en la capacidad para lograr retener nueva información (Botwinick, 1978; Craik, 1977; Hulicka y Weiss, 1965; citados en Riquelme et al., 2010, p. 283) y en una mayor afectación en la memoria episódica (Nilsson, 2003; Nilsson 2004; citados en Correira, 2011, p. 7).

Por otro lado, se utiliza frecuentemente el término funciones ejecutivas (FE) para referirnos a una serie de funciones cognitivas superiores llevadas a cabo por un sistema mental de alta complejidad, cuya base neurológica se encuentra en el córtex prefrontal (cíngulo y zonas neocorticales anteriores). Algunos de los trabajos llevados a 
cabo por las funciones ejecutivas son los siguientes: planificación de acciones no rutinarias, planificación de estrategias, aprendizaje intencional, inhibición de estímulos distractores, resolución de conflictos mentales, flexibilidad, etc. Es decir, a través de las funciones ejecutivas llevamos a cabo los comportamientos premeditados y autocontrolados que nos permiten adaptarnos a nuevas situaciones.

Pues bien, es importante destacar que los lóbulos frontales y las funciones ejecutivas parecen ser más vulnerables al envejecimiento normal que otras regiones del cerebro. Por ello, suelen ser estos procesos los que primero sufren deterioro cognitivo asociado a la edad (Froufe, Cruz, y Sierra, 2009). Según diversos estudios clínicos con ancianos con envejecimiento normal, el decremento que se produce en las funciones ejecutivas incluye el pensamiento abstracto, la flexibilidad mental y la capacidad para responder a los cambios del entorno, entre otros aspectos (Iavarone et al., 2011).

Una vez expuestos los principales argumentos teóricos, el objetivo de esta investigación es realizar un estudio sobre el estado de las funciones cognitivas en el envejecimiento, más concretamente, el estado de la memoria y las funciones ejecutivas. En este sentido, uno de los propósitos de la presente investigación es comparar grupos de ancianos con diferentes niveles de cognición (con deterioro cognitivo leve y sin deterioro), así como comparar los datos obtenidos en ambas muestras de sujetos (residentes y no residentes); y además, analizar la repercusión que dichos niveles tienen con respecto a las puntuaciones obtenidas en memoria y funciones ejecutivas en sus respectivas pruebas. De este modo, las hipótesis planteadas para poner a prueba son las siguientes: 1) a mayor nivel de deterioro cognitivo, menor rendimiento en prueba de memoria (T@M); 2) a mayor nivel de deterioro cognitivo, menor rendimiento en prueba de funciones ejecutivas (Test de las Anillas); 3) existen diferencias significativas entre la variable "nivel de deterioro cognitivo" y los resultados obtenidos en memoria y funciones ejecutivas; 4) no existen diferencias significativas entre la variable "sexo" y los resultados obtenidos en memoria y funciones ejecutivas y 5) no existen diferencias significativas entre la variable "residencia" y los resultados obtenidos en memoria y funciones ejecutivas.

\section{MÉTODO}

\section{Participantes}

El tipo de muestreo utilizado en este estudio fue muestreo intencional. La muestra de participantes fue seleccionada de forma voluntaria en una residencia de ancianos (50\% de participantes) y fuera de ella (50\% de participantes). Con respecto a los criterios de inclusión, hemos de decir que, se seleccionaron, para un grupo (Deterioro Cognitivo Leve), aquellos participantes que puntuaron por debajo del punto de corte establecido para detectar el deterioro cognitivo según el Mini-Mental State Examination 
(MMSE) de Folstein, Folstein, y McHugh (1975) y por otro lado, para el otro grupo (no Deterioro Cognitivo), aquellos que puntuaron por encima de dicho punto de corte, el cual se sitúa en 27 y 23 puntos para personas no institucionalizadas y personas institucionalizadas, respectivamente; y además, se estableció como criterio no tener diagnóstico o antecedente de enfermedad neurológica ni psiquiátrica.

Teniendo en cuenta estos criterios, participaron un total de 24 sujetos. Con respecto al sexo había el 50\% $(N=12)$ de mujeres y el 50\% $(N=12)$ de hombres. La media de edad fue de 78 años con una desviación típica de 7.388.

\section{Instrumentos}

Se administraron tres instrumentos para recabar información sobre el deterioro cognitivo, las funciones ejecutivas y la memoria. A continuación se describen brevemente los instrumentos utilizados:

-Mini-Examen Cognoscitivo (MEC-35; Lobo, Ezquerra, Gómez, Sala, y Seva, 1979), que es la adaptación española del Mini-Mental State Examination (MMSE) de Folstein, et al. (1975). Es un test de cribado que suele utilizarse con frecuencia para detectar el deterioro cognitivo. Examina de forma rápida un conjunto de funciones cognitivas que pueden encontrarse afectadas en población anciana (orientación, memoria, atención y cálculo, lenguaje y construcción, praxias y razonamiento). La puntuación que se obtiene en esta prueba suele utilizarse como índice global del estado del funcionamiento cognitivo de la persona en cuestión. El punto de corte se sitúa, para personas mayores de 65 años y con un nivel educativo medio, en 27 y 23 puntos de un total de 35, para no geriátricos y geriátricos, respectivamente.

- Test de las Anillas (Portellano y Martínez-Arias, 2011). Prueba de cribado que evalúa el funcionamiento ejecutivo mediante la capacidad de planificación, por ser ésta, uno de los componentes esenciales y más sensibles de las funciones ejecutivas. Consiste en 15 ítems de dificultad creciente en los que el sujeto, moviendo una a una las anillas colocadas en un tablero con tres postes, debe reproducir un modelo presentado en una lámina. El tiempo de aplicación estimado, es de, aproximadamente, 15 minutos.

-Test de Alteración de Memoria -T@M- (Rami, Molinuevo, Bosch, Sánchez-Valle, y Villar, 2007). Se trata de un test de cribado que consta de 50 ítems. Evalúa varios subtipos de memoria (memoria inmediata, memoria de orientación temporal, memoria remota semántica, memoria de evocación libre y memoria de evocación con pistas). A la hora de clasificar a los participantes, se utiliza un punto de corte de 37 para diferenciar deterioro cognitivo de quejas subjetivas de memoria.

\section{Procedimiento}

Los participantes fueron evaluados en dos contextos de referencia: sus propios hogares y una Residencia de Ancianos (Lorca, Murcia). En la Residencia de Ancianos, la 
selección de los participantes se llevó a cabo con el permiso de la Dirección del centro y con la ayuda de la psicóloga del mismo, la cual proporcionó la información previa sobre aquellos residentes que podían ser considerados como personas con o sin deterioro cognitivo, y a los cuales se les podría llevar a cabo una evaluación de dichas capacidades. Por otro lado, aquellos ancianos que fueron evaluados en sus hogares, fueron escogidos en función de las puntuaciones obtenidas en la prueba MEC-35 para ser asignados al grupo con deterioro cognitivo leve o sin deterioro cognitivo. Pues bien, todos los sujetos participaron de forma voluntaria y fueron debidamente informados del objeto de la investigación. Además, los cuestionarios fueron administrados por uno de los autores del estudio durante los meses de febrero y abril del año 2014.

Podríamos decir que la investigación se dividió en dos fases. Primera fase: los participantes fueron clasificados en uno de los dos grupos (personas con o sin deterioro cognitivo) a partir de la puntuación obtenida en el "Mini-Examen Cognoscitivo MEC35". Una vez realizados ambos grupos, se llevó a cabo la segunda fase: administración de los cuestionarios de memoria y funciones ejecutivas (T@M y Test de las Anillas, respectivamente). Una vez administradas todas las pruebas necesarias a los participantes de la investigación, se pasó al análisis de los datos obtenidos y a su interpretación.

\section{Análisis de datos}

Para esta parte de la investigación, el tratamiento estadístico de los datos, se utilizó el programa estadístico informático SPSS, en su versión 19.

Por un lado, y en lo que respecta al análisis de los datos descriptivos, éste se realizó utilizando el estudio de frecuencias en media y desviación típica. Por otro lado, para el análisis de las variables cuantitativas y las variables categóricas, se utilizó la prueba $t$ de Student para muestras independientes, llevando a cabo un análisis de diferencias de medias. Además de ello, se comprobó previamente el supuesto de homogeneidad de varianzas de Levene.

Finalmente, en cuanto al análisis de las variables cuantitativas, se utilizó el coeficiente de correlación de Pearson, realizando un análisis de correlaciones bivariadas.

\section{RESULTADOS}

En lo que respecta a las dos primeras hipótesis, se realizó un análisis de correlaciones bivariadas a través del coeficiente de correlación de Pearson para comprobar la relación existente entre nivel de deterioro cognitivo y memoria, y entre nivel de deterioro cognitivo y funciones ejecutivas (para hipótesis 1 y 2, respectivamente). Por un lado, para la hipótesis número 1 se ha encontrado una correlación de Pearson significativa positiva de magnitud elevada entre dichas variables 
(Figura 1); lo que significa que, conforme aumenta el buen estado de las funciones cognitivas, aumenta el rendimiento en memoria, $(r=.777 ; p=.000 ; N=24)$.

Figura 1. Gráfico de dispersión para memoria y estado cognitivo

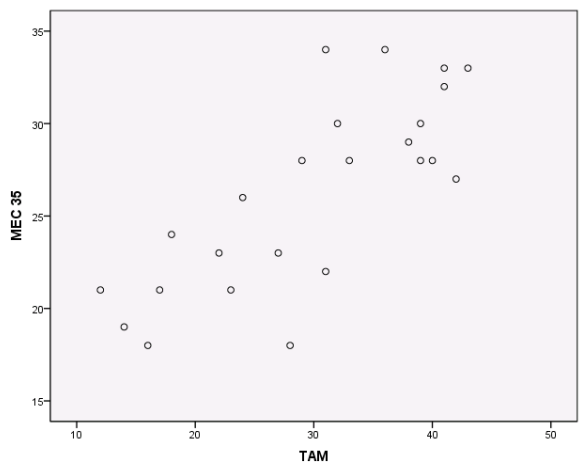

Por otro lado, para la hipótesis número 2 se ha encontrado una correlación de Pearson significativa negativa de magnitud moderada entre nivel de deterioro cognitivo y funciones ejecutivas (Figura 2); lo que significa que, conforme aumenta el buen estado de las funciones cognitivas, disminuye el tiempo de ejecución en el Test de las Anillas, lo que es indicativo de un mejor funcionamiento ejecutivo, $(r=-.574 ; p=.003 ; N=24)$.

Figura 2. Gráfico de dispersión para funciones ejecutivas y estado cognitivo

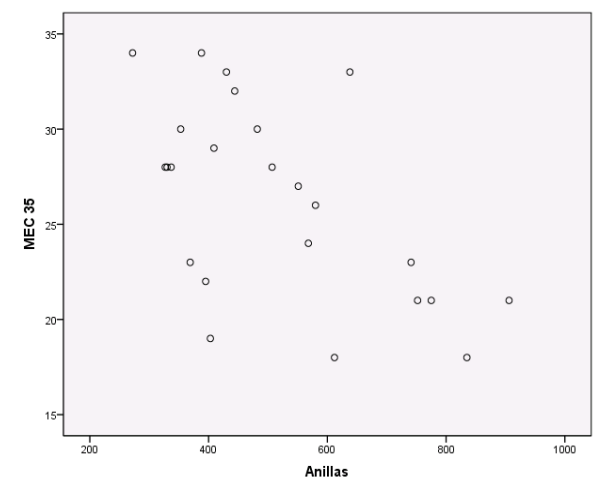

Finalmente, para las hipótesis 3, 4 y 5, se realizó la prueba t de Student para muestras independientes. Por un lado, para la hipótesis número 3 , atendiendo al número total de participantes, en cuanto al nivel de deterioro cognitivo aparecieron diferencias de medias significativas, asumiendo varianzas iguales, entre el deterioro cognitivo y las variables memoria $y$ funciones ejecutivas, siendo respectivamente $t_{(22)}=-5.068$, 
$p=.000$ y $t_{(17.215)}=3.615, p=.002$. En el primer caso, la media de las personas sin deterioro fue superior a la media de las personas con deterioro (Figura 3); en el segundo caso, la media de las personas con deterioro fue superior a la media de las personas sin deterioro (Figura 4).

Figura 3. Medias según deterioro cognitivo en memoria

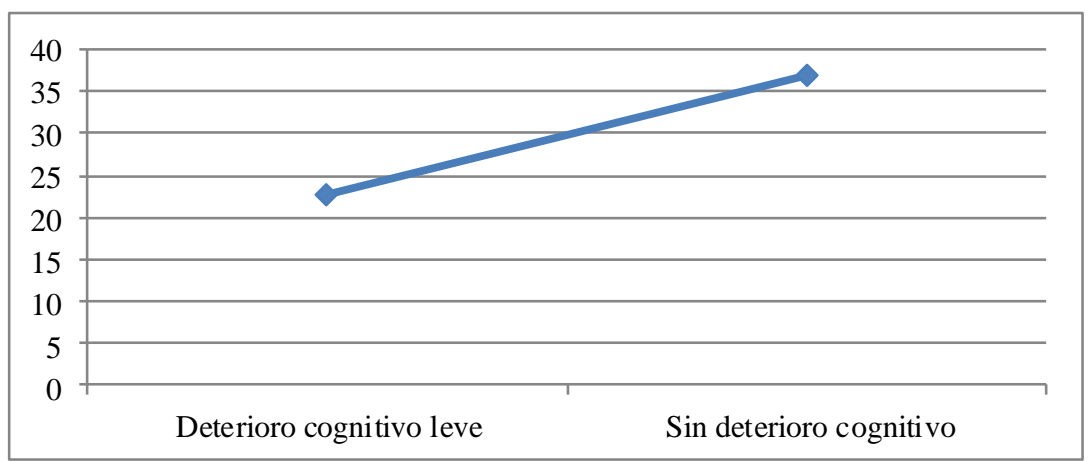

Figura 4. Medias según deterioro cognitivo en funciones ejecutivas

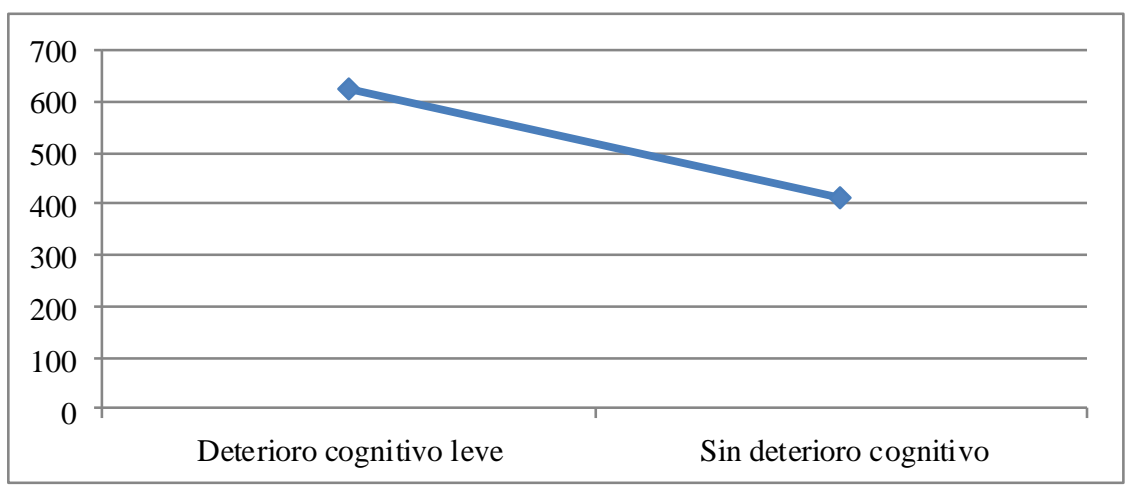

Pues bien, las puntuaciones medias para la variable memoria resultaron significativamente más elevadas para el grupo de personas sin deterioro cognitivo debido al mayor número de ítems correctos en el test que medía dicha capacidad. Y, por el contrario, las puntuaciones medias para la variable funciones ejecutivas resultaron significativamente menores para el grupo de personas sin deterioro cognitivo debido al menor tiempo de ejecución empleado en la prueba que medía esta capacidad.

Por otro lado, para la hipótesis número 4, atendiendo al número total de participantes, en cuanto al sexo no se obtuvieron diferencias de medias significativas en la prueba $t$ de Student y la variable memoria. Si bien es cierto que aparecieron 
diferencias de medias significativas, asumiendo varianzas iguales, entre el sexo y la variable funciones ejecutivas, siendo $t_{(16.056)}=2.293, p=.036$. La media de los hombres fue superior a la media de las mujeres (Figura 5).

Figura 5. Medias según sexo en el Test de las Anillas

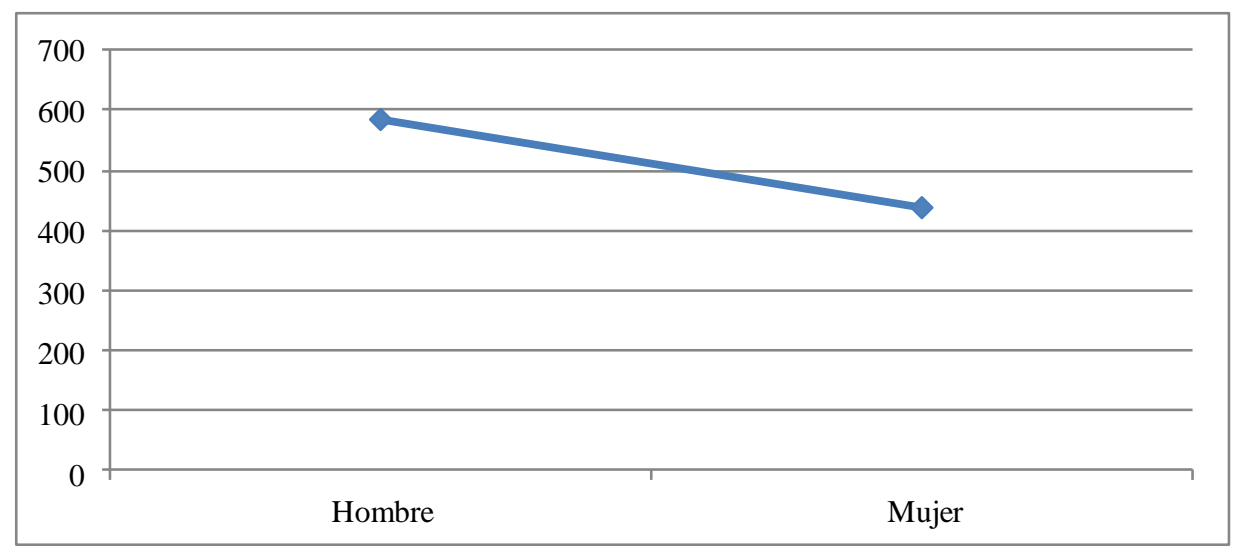

Y finalmente, para la hipótesis número 5, atendiendo al número total de participantes, en cuanto al lugar de residencia no se obtuvieron diferencias de medias significativas en la prueba $t$ de Student y las variables memoria y funciones ejecutivas, siendo respectivamente $t_{(22)}=-.832, p=.414$ y $t_{(17.177)}=1.397, p=.180$.

\section{DISCUSIÓN}

Este estudio ofrece resultados en base a las diferencias existentes en sujetos ancianos con y sin deterioro cognitivo y el estado de la memoria y las funciones ejecutivas de los mismos. De este modo, por un lado se ha podido confirmar la estrecha relación existente entre nivel de deterioro cognitivo y memoria, lo cual se encuentra en la línea de lo que pretendíamos comprobar. A este respecto, los resultados se encuentran en concordancia con lo aportado por diversos autores, como González et al. (2001), Aller (2002), Ventura (2004) y Montes-Rojas et al. (2012), que indican que el envejecimiento conlleva una pérdida cognitiva que refleja diversos cambios, los cuales provocan un descenso en memoria, velocidad de procesamiento, concentración, etc.

Por otro lado, nuestros resultados determinan la relación existente entre nivel de deterioro cognitivo y funciones ejecutivas, con lo cual, a medida que aumenta el nivel de deterioro cognitivo, aumenta la dificultad para llevar a cabo un proceso de planificación de acciones para solucionar un determinado problema; este hecho permite confirmar una de las hipótesis establecidas en este estudio. Dichos resultados se 
encuentran en concordancia con lo aportado por autores como Binotti, Spina, de la Barrera, y Donolo (2009), que afirman los cambios sufridos por las funciones ejecutivas con el paso de los años; Froufe et al. (2009), que afirman que los jóvenes utilizan con más frecuencia la estrategia y planificación que las personas mayores con algún tipo de deterioro, además de que las personas adultas sanas actúan de forma estratégica, a diferencia de aquellas con deterioro cognitivo; y Aller (2002), que mantiene que el deterioro asociado al lóbulo frontal se traduce en una habilidad reducida para la planificación.

Así mismo, encontramos un dato curioso en lo relativo a las diferencias de género y nuestras variables dependientes (memoria y funciones ejecutivas). Se ha podido confirmar que no existen diferencias significativas en cuanto a las variables sexo y memoria. No obstante, es cierto que se obtuvieron diferencias significativas en cuanto a las variables sexo y funciones ejecutivas. Este último hallazgo se aleja de lo esperado, puesto que lo predecible era no obtener diferencias, del mismo modo que en cuanto a sexo y memoria; lo cual parece indicar que las diferencias de género se hacen más evidentes en determinadas actividades, como puede ser la planificación de acciones. Aunque bien es cierto, tal y como indican Navarro, Calero, y Calero-García (2014), que podrían existir variables no controladas que sean las causantes de las diferencias halladas

$\mathrm{Y}$ por último, se ha podido comprobar que no existen diferencias significativas en cuanto a las variables residencia y funciones cognitivas (memoria y funciones ejecutivas); con lo cual podemos afirmar, en concordancia a la última hipótesis establecida, que no existe relación entre la pertenencia o no a una residencia de personas mayores y el estado de las funciones cognitivas.

De este modo, podríamos concluir que los resultados obtenidos en este estudio confirman el hecho de que el aumento del deterioro cognitivo afecta considerablemente a las funciones mentales y al intelecto.

\section{REFERENCIAS}

Aller, N. (2002). Aspectos neuropsicológicos del envejecimiento cerebral normal y patológico. Gabinete de servicios sociales ANATER.

Binotti, P., Spina, D., de la Barrera, M.L., y Donolo, D. (2009). Funciones ejecutivas y aprendizaje en el envejecimiento normal. Estimulación cognitiva desde una mirada psicopedagógica. Revista Chilena de Neuropsicología, 4(2), 119-126. Recuperado de http://www.neurociencia.cl/dinamicos/articulos/804786-rcnp2009v4n2-6.pdf

Correia, R. (2011). Cambios cognitivos en el envejecimiento normal: influencias de la edad y su relación con el nivel cultural y el sexo. (Tesis doctoral). Universidad de la Laguna, La Laguna. Recuperado de ftp://tesis.bbtk.ull.es/ccssyhum/cs475.pdf

Folstein, M.F., Folstein, S.E., y McHugh, P.R. (1975). "Mini-Mental State": A practical method for grading the cognitive state of patients for the clinician. Journal of Psychiatric Research, 12, 397-408. 
Froufe, M., Cruz, I., y Sierra, B. (2009). (dis)Función ejecutiva en personas mayores con y sin Alzheimer: Actuación estratégica basada en expectativas. Psicológica, 30, 119-135. Recuperado de http://www.uv.es/revispsi/articulos1.09/6FROUFE.pdf

García-Sevilla, J., Fernández, P.J., Fuentes, L.J., López, J.J., y Moreno, M.J. (2014). Estudio comparativo de dos programas de entrenamiento de la memoria en personas mayores con quejas subjetivas de memoria: un análisis preliminar. Anales de psicología, 30(1), 337-345. Recuperado de http://revistas.um.es/analesps/article/view/analesps.30.1.1 $58021 / 156601$

González, R., Zapata, J.A., Pérez, J.C., Hormigo, A., Guerrero, R.D., y Baca, A. (2001). Estudio del déficit cognitivo en pacientes ingresados en una residencia geriátrica. Medicina General, 38, 792-796.

Iavarone, A., Lorè, E., De Falco, C., Milan, G., Mosca, R., Pappatà, S., ... Postiglione, A. (2011). Dysexecutive performance of healthy oldest old subjects on the Frontal Assessment Battery. Aging Clinical And Experimental Research, 23(5-6), 351-356. doi:10.3275/7809

Instituto Nacional de Estadística (INE). (2014). España en cifras 2014. Recuperado de http://www.ine.es/prodyser/espa_cifras/2014/

Lobo, A., Ezquerra, J., Gómez, F., Sala, J.M., y Seva, A. (1979). El mini-examen cognoscitivo. Un test sencillo y práctico para detectar alteraciones intelectuales en pacientes médicos. Actas Luso Españolas de Neurología y Psiquiatría, 7, 189-201

Montes-Rojas, J., Gutiérrez-Gutiérrez, L., Silva-Pereira, J.F., García-Ramos, G., y del RíoPortilla, Y. (2012). Perfil cognoscitivo de adultos mayores de 60 años con y sin deterioro cognitivo. Revista Chilena de Neuropsicología, 7(3), 121-126. doi:10.5839/rcnp.2012.0703.05

Navarro, E., Calero, M.D., y Calero-García, M.J. (2014). Diferencias entre hombres y mujeres mayores en funcionamiento cognitivo y calidad de vida. European Journal of Investigation in Health, Psychology and Education, 4(3), 267-277. doi: 10.1989/ ejihpe.v4i3.74

Portellano, J.A., y Martínez-Arias, R. (2011). Anillas: Test para la Evaluación de las Funciones Ejecutivas. Madrid: TEA Ediciones.

Rami, L., Molinuevo, J. L., Bosch, B., Sánchez-Valle, R., y Villar, A. (2007). Hospital Clínic i Universitari de Barcelona. T@M - Test de Alteración de Memoria. Recuperado de http://www.recorda.info/u/uploads/File/TM.pdf

Riquelme, A., Ortigosa, J.M., y Martín, M. (Coords.). (2010). Manual de Psicogerontología. Madrid: Ediciones Académicas, S. A.

Rodríguez-Aranda, C., y Sundet, K. (2006). The Frontal Hypothesis of Cognitive Aging: Factor Structure and Age Effects on Four "Frontal Tests" among Healthy Individuals. Journal of Genetic Psychology, 167(3), 269-287. Recuperado de http://web.a.ebscohost.com /ehost/detail?vid=30\&sid=4d02d0e7-4c9f-460a-9ab09d139cc8f290\%40sessionmgr400 $3 \&$ hid $=4104 \&$ bdata $=$ Jmxhbmc9ZXMmc210ZT1laG9zdC1saXZl\#db=eric\&AN=EJ8768 20

Ventura, R. (2004). Deterioro cognitivo en el envejecimiento normal. Revista de psiquiatría y salud mental Hermilio Valdizan, 5(2), 17-25. Recuperado de http://www.hhv.gob pe/revista.html

Recibido: 2 de junio de 2015 Recepción Modificaciones: 31 de julio de 2015 Aceptado: 2 de agosto de 2015 